\title{
ON IMPROVING EFFICIENCY OF DIFFERENTIAL EVOLUTION FOR AERODYNAMIC SHAPE OPTIMIZATION APPLICATIONS
}

\author{
Nateri K. Madavan ${ }^{*}$ \\ NASA Ames Research Center, Moffett Field, California
}

\begin{abstract}
Differential Evolution (DE) is a simple and robust evolutionary strategy that has been proven effective in determining the global optimum for several difficult optimization problems. Although DE offers several advantages over traditional optimization approaches, its use in applications such as aerodynamic shape optimization where the objective function evaluations are computationally expensive is limited by the large number of function evaluations often required. In this paper various approaches for improving the efficiency of $\mathrm{DE}$ are reviewed and discussed. Several approaches that have proven effective for other evolutionary algorithms are modified and implemented in a DE-based aerodynamic shape optimization method that uses a Navier-Stokes solver for the objective function evaluations. Parallelization techniques on distributed computers are used to reduce turnaround times. Results are presented for standard test optimization problems and for the inverse design of a turbine airfoil. The efficiency improvements achieved by the different approaches are evaluated and compared.
\end{abstract}

\section{INTRODUCTION}

Aerodynamic shape optimization refers to the process of determining the shapes of airfoils, wings, or other aerodynamic surfaces that are optimal with regard to one or several desired characteristics. Major advances in the field of aerodynamic shape optimization have been achieved in recent years by combining improved methods for the simulation of complicated flow fields with efficient numerical optimization techniques and by exploiting the powerful capabilities of modern computers. Both Euler and high-fidelity Navier-Stokes solvers have been combined with various traditional optimization techniques (gradient-based methods, response surfaces, etc.) as well as non-traditional approaches such as neural networks and evolutionary algorithms to obtain optimal aerodynamic shapes and designs.

* Research Scientist, NASA Advanced Supercomputing Division. Senior Member, AIAA.
This article deals with an evolutionary algorithm (EA) known at Differential Evolution ${ }^{1,2}$ for aerodynamic shape optimization. DE is a simple and robust evolutionary strategy (ES) that has been proven effective for several difficult optimization problems. ${ }^{3}$ Its application in aeronautics, ${ }^{4,5,6,7,8,9}$ however, has been somewhat limited. Generally speaking, population-based evolutionary approaches such as $\mathrm{DE}$ are easy to implement and are quite robust and capable of locating the global optimum. However, as with other EA approaches, DE often require a lot of objective function evaluations to arrive at the optimal solution. This poses a serious impediment to the use of DE in aerodynamic shape optimization applications where the objective function evaluations are performed by computationally expensive analysis codes based on the Euler or Navier-Stokes equations.

The purpose of this article is to explore and summarize various modifications to the $\mathrm{DE}$ algorithm that can lead to improved computational efficiency and apply them to aerodynamic shape optimization problems. Several promising approaches that have proven effective in the context of other EAs have been modified and implemented in a DE-based aerodynamic shape optimization method that uses a Navier-Stokes solver for objective function evaluations. The shape optimization method is implemented on distributed parallel computers so that new designs can be obtained within reasonable turnaround times. Results are presented for standard test optimization problems from the literature and for the inverse design of a turbine airfoil. The efficiency improvements achieved by the different approaches are evaluated and compared. Some of these approaches have been reported by the author in earlier work. ${ }^{8,9}$ This article extends this prior work and summarizes our efforts to date aimed at improving the efficiency of the $\mathrm{DE}$ algorithm.

Other efforts to improve the efficiency of the DE algorithm have also been reported in the literature. Chiou and Wang ${ }^{10}$ developed a hybrid $\mathrm{DE}$ algorithm that incorporated a gradient-based local search method for chemical engineering applications. In aeronautics, Rogalsky et al. ${ }^{6}$ developed a DE hybrid by incorporating a Nelder-Mead downhill simplex search and used it with a potential flow solver in the inverse design of turboma- 
chinery airfoils. Airfoil design optimization methods that use the DE algorithm in conjunction with neural network strategies have also been reported by Rai. ${ }^{7}$

\section{DIFFERENTIAL EVOLUTION}

$\mathrm{DE}$ is a conceptually simple ES developed for singleobjective optimization in continuous search spaces with good convergence properties that have been demonstrated in a variety of applications. ${ }^{3}$ Details of the algorithm can be found elsewhere; ${ }^{1,2}$ only its main features are summarized here.

DE uses a population $P$ that contains $m n$-dimensional real-valued parameter vectors, where $n$ is the number of parameters or decision variables:

$$
P=\left\{\bar{x}_{1}, \ldots, \bar{x}_{m}\right\}
$$

The population is usually initialized at generation $g=0$ in a random fashion:

$$
P(0)=\left\{\bar{x}_{1}(0), \ldots, \bar{x}_{m}(0)\right\}, g=0
$$

The population size $m$ is maintained constant throughout the optimization process. Differential evolution is thus similar to a $(\mu, \lambda) \mathrm{ES}^{11}$ with $\mu$ and $\lambda$ equal to $m .{ }^{12}$ The method however differs from standard ES approaches in several respects as described below.

As with all ES-based approaches, mutation is the key ingredient of differential evolution. The basic idea is to generate new parameter vectors for the subsequent generation by using weighted differences between two (or more) parameter vectors selected randomly from the current population to provide appropriately scaled perturbations that modify another parameter vector (or, comparison vector) selected from the same population. This idea has been implemented in various forms. In the the classical implementation that is rather popular, new trial parameter vectors $\left\{\bar{y}_{1}, \ldots, \bar{y}_{m}\right\}$ for the next generation $g+1$ are generated according to the following mutation scheme:

For $l=1, m ; i=1, n$ generate

$$
y_{l}^{i}=x_{\alpha_{1}^{l}}^{i}(g)+F \cdot\left(x_{\alpha_{2}^{l}}^{i}(g)-x_{\alpha_{3}^{l}}^{i}(g)\right)
$$

In the above $\alpha_{1}^{l}, \alpha_{2}^{l}, \alpha_{3}^{l}$ are distinct elements of $\{1,2, \ldots, \mathrm{m}\}$ randomly selected for each $l$, and $F \in[0,2]$ is a parameter that controls the amplification of the differential variation. Other variants that either use the difference between more than two parameter vectors or keep track of the best parameter vector at each generation and use it in the mutation scheme have also been developed ${ }^{1}$ and used with varying success in specific applications.

DE is similar to other recombinant ES approaches in that it also uses discrete recombination. The strategy adopted in differential evolution is to modify the trial parameter vectors $\left\{\bar{y}_{1}, \ldots, \bar{y}_{m}\right\}$ to generate parameter vectors $\left\{\bar{z}_{1}, \ldots, \bar{z}_{m}\right\}$ as follows:

For $l=1, m ; i=1, n$ generate

$$
z_{l}^{i}=\left(\begin{array}{c}
y_{l}^{i} \text { with probability } p_{c} \\
x_{l}^{i}(g) \text { with probability } 1-p_{c}
\end{array}\right.
$$

where $p_{c}$ is a parameter that controls the proportion of perturbed elements in the new population. Note that the mutation and recombination operations described above can lead to new vectors that may fall outside the boundaries of the variables.. Various repair rules can be used to ensure that these inadmissible vectors do not enter the population. A simple strategy, which is the one adopted here, is to delete these inadmissible vectors and form new ones until the population is filled.

The selection scheme used in DE is deterministic but differs from methods usually employed in standard ES approaches. Selection is based on local competition only, with the modified trial parameter vector competing against one population member (the comparison vector) and the survivor entering the new population $g+1$ as follows:

$$
\begin{aligned}
& \text { For } l=1, m \\
& \bar{x}_{l}(g+1)=\left(\begin{array}{lll}
\bar{z}_{l} & \text { if } & f\left(z_{l}\right)<f\left(x_{l}(g)\right) \\
\bar{x}_{l}(g) & \text { else }
\end{array}\right.
\end{aligned}
$$

where $f$ denotes the corresponding objective function (or fitness) value. This greedy selection criterion results in fast convergence; the adaptive nature of the mutation operator, in general, helps safeguard against premature convergence and allows the process to extricate itself from local optima. The generation counter is incremented and the process is repeated until some stopping criteria are satisfied.

\section{EFFICIENCY IMPROVEMENT STRATEGIES FOR DIFFERENTIAL EVOLUTION}

A variety of approaches that seek to improve the computational efficiency of evolutionary approaches without compromising their desirable features have been proposed in the literature. Several of these were evaluated in the present study in the context of the Differential Evolution algorithm and are discussed below.

\section{Metamodeling Techniques}

Metamodeling techniques for improving the efficiency of evolutionary approaches are based on the use of approximate models as surrogates for the actual objective functions. These surrogates can be incorpo- 
rated in the optimization process and their judicious use can reduce the number of calls to the expensive analysis codes. One metamodeling approach that has received much attention is the response surface method (RSM). While traditional RSM uses low-order polynomials for function approximation, generalized response surface methods (GRSM) allow for the inclusion of a wide range of approximations, including polynomials, neural networks, kriging, multivariate adaptive regression splines, radial basis functions, and multiquadrics. Both global and local GRSM approaches have been established. In the global approach a GRSM metamodel for the entire design space is used and gradually refined as the optimization progresses. Since developing good global metamodels with validity over the entire design space can be difficult, a local approach based on local approximations and a sequential approximate optimization strategy for iteratively zooming into the region of design space around the optimum is often preferred. Typically, the optimization process is decomposed into a sequence of cycles and an optimization subproblem is defined within a trust region, i.e., a smaller part of the design space, where local metamodels are used as surrogates for the exact objective functions. The exact objective functions are evaluated only at a limited number of points in each trust region thus reducing computational cost. The trust regions are resized and/or moved as the optimization progresses. Various optimization frameworks based on such trust-region and move-limit methods have been developed to strike an appropriate balance between the use of exact and approximate function evaluations. ${ }^{13}$

The GRSM metamodeling strategy for DE of Madavan $^{8}$ with metamodels based on artificial neural networks is used here. A simple strategy is adopted where the metamodel is used only in the latter stages of the optimization after the population has evolved to the general vicinity of the optimal solution. This eliminates the need for a more elaborate sequential zooming strategy although it limits the efficiency improvements. Using the neural network as a local response surface with validity only in a small region of the design space makes neural network training easier and improves network generalization abilities. A three-layer feed-forward neural network is used here.

\section{Memetic Methods}

Generally speaking, evolutionary algorithms are not well suited for fine-tuned search close to the optimal solution. Another approach to improving the efficiency of evolutionary approaches is the use of memetic methods that apply a separate local search process to refine specific individuals. These methods are also referred to as hybrid algorithms or genetic local searchers. The general idea is to combine the global nature of evolutionary search with more efficient deterministic local search techniques. A simple strategy often adopted is to perform a two-stage optimization with the best solution determined by the GA being used as the starting point for the local search method. Alternatively, strategies that permit dynamic coupling and interaction between the GA and the local search method can be used that intuitively seem likely to be more effective.

In the context of $\mathrm{DE}$, memetic methods have been explored in Madavan ${ }^{9}$ where the DE algorithm. was combined with a dynamic hill climbing 14,15 (DHC) local search technique in order to exploit the complementary advantages of both methods and achieve better computational efficiency than standard DE. This approach is used here and described briefly below.

The core of the basic DHC algorithm is an efficient technique for locating local optima. ${ }^{14}$ The search originates at a specified location given by the vector $\bar{x}$ of the design parameters and uses a probing vector $\bar{v}$ whose length is initially specified but increases or decreases dynamically to suit the local objective function terrain. Random move directions are tried (up to a specified maximum number) for a given probing vector length $|v|$. If the objective function value at a new point, $\bar{x}+\bar{v}$, is better than the previous value, the probing vector length is doubled and further regions of the domain are searched; if not, the vector length is halved and a more localized search is performed. In addition, a memory vector $\bar{u}$ is also used to keep track of the previous successful move direction. A linear combination $\bar{u}+\bar{v}$ of this vector and the probing vector is also evaluated as a candidate move direction. The process stops when the probing vector length falls below a specified (small) threshold value.

An important decision that has to be made for effcient optimization is the relative effort levels for the global and the local search. There are various aspects to this decision dealing with which and how many population members should be chosen for the local search, when the local search should be invoked, and when it should be terminated. The standard approach is to specify these parameters and keep their values fixed during the optimization. However, this involves considerable parameter tuning to obtain the best values for a particular application. Further, the tuning process is application-specific and will have to be repeated for a different application. Following Espinoza, et al. ${ }^{16}$ an alternate approach is used in Madavan ${ }^{9}$ where these parameters are not specified but adapt in response to recent performance as the algorithm converges to the optimal solution. The change in the relative coefficient of variation (CV) of the fitness 
function between generations is monitored. ${ }^{16} \mathrm{CV}$ is defined as the ratio of the mean and the standard deviation of the population fitness. In a similar fashion, the number of local search iterations to be performed before switching back to the global DE search is decided by comparing the most recent fitness improvement by local search with the latest fitness improvement by global search. ${ }^{16}$ In general, local search typically is performed on a small subset of the population and the probability of a population member being selected for local search can also be made to adapt with the solution. ${ }^{16}$ However, in the results reported here, only one (either the current best or randomly chosen) member of the population from a generation is selected for the local search.

\section{Subpopulation or Island Techniques}

Another efficiency enhancement technique that has had some success in certain applications is the use of subpopulation or island genetic algorithms. ${ }^{17,18}$ These are essentially coarse-grained distributed genetic algorithms where multiple subpopulations or "islands" are evolved in a distributed fashion with interaction occurring between the islands through occasional migration of individuals. Each island searches its own space for feasible solutions. The islands can be connected through various topologies: bidirectional rings, unidirectional rings, hypercubes, etc. Different parameter settings and fitness function models of varying fidelity or resolution can be used in the various islands. For example, there can be several islands using inexpensive low-fidelity evaluations that progressively pass on individuals to fewer islands using higher fidelity evaluations. The final paper will discuss the present implementation in more detail.

\section{Adaptive Parameter Range Techniques}

The idea in adaptive parameter range techniques is to dynamically alter the parameter range of the space being searched. The idea originated with binary-coded genetic algorithms and is also referred to as dynamic coding ${ }^{19}$ or stochastic genetic algorithm. ${ }^{20}$ With dynamic coding higher precision is achieved with the same chromosome length as the extent of the search space is gradually refined during the optimization process. These dynamic coding ideas have also been extended to real-coded genetic algorithms. The approach implemented here in the DE algorithm closely follows that of Oyama et al. ${ }^{21}$ More details will be provided in the final paper.

\section{Other Associated Strategies}

Some additional improvement strategies that have been incorporated here to enhance the efficiency of the DE algorithm are described in this subsection. It is noted that these have been used both individually or in combi- nation with each other and in some fashion with all of the broad techniques described above.

\section{Variable Complexity Strategies}

The main idea in variable complexity modeling strategies 22 is to tailor the "complexity" of the analysis codes used in objective function evaluation to meet the needs of the optimization phase or method as it evolves toward the optimum solution. This is also referred to as multiscale or multilevel strategies in the literature. In the context of aerodynamic design, "complexity" can mean any of several attributes of the CFD analysis codes used, such as fidelity level, grid density, and convergence criteria. Variable complexity modeling is implemented here by using low fidelity, coarse grid Euler computations in the initial phases of the DE optimization followed by fine-grid Navier-Stokes analyses that are more appropriate as the algorithm approaches convergence. One crucial issue in using information from numerical models of varying fidelity on varying grid sizes is that the numerical inaccuracies can impart variation in the fitness function evaluations for the same candidate designs. However, the sequential strategy used here works well for the problems considered.

\section{Small Population or Micro-GA Strategies}

Another strategy for improving efficiency is to reduce the overall population size. This must be done with care to avoid premature convergence and not sacrificing robustness or reliability. Although more generations are typically required for convergence, the overall number of objective function evaluations required can be smaller since fewer evaluations are being.performed at each generation.

In order to make the DE algorithm work reliably with much smaller population sizes a diversity-preserving technique is incorporated. When using DE in function optimization, an acceptable trade-off between convergence and population diversity must be determined. The DE parameters $F$ and $p_{c}$ can be adjusted to control the convergence speed but population diversity is a requisite for ensuring that the algorithm converges to a global, rather than local, optimum. Diversity is a function of the population size, and for a given problem, DE typically becomes more robust with convergence speeds that are less sensitive to the choice of $F$ and $p_{c}$ as the population size is increased. However, when DE is used with small population sizes, the population can lose diversity and get closely clustered. The DE mutation and crossover operations are then unable to generate better new individuals and the algorithm converges prematurely to a local optimum and population diversity is lost as all members of the population soon cluster around this point. In order to maintain population diversity, a degree 
of diversity parameter ${ }^{10}$ is monitored in the current approach. If this measure of diversity falls below a specific tolerance level, only the current best population member is retained and the remaining population is reinitialized for the next generation. While frequent population reinitialization slows down convergence, results show that it is possible to achieve reductions in the overall number of function evaluations by using a much smaller population size than what would be normally required to maintain diversity and converge reliably to the global optimum.

\section{Function Evaluation Reuse Strategies}

This is a simple idea of storing all the evaluated solutions and their fitness values in a database for later reuse during the optimization process. This approach can save a significant amount of computation time. In particular, instead of initializing a new Navier-Stokes evaluation from freestream conditions the solution from the nearest (in an Euclidean sense) design in the database can be used. as the starting solution.

\section{Parallel Computing Strategies}

The discussion so far has centered on the enhancing algorithmic efficiency of $\mathrm{DE}$ to improve overall design cycle time and cost. In addition, substantial reductions in overall design time can also be achieved by resorting to parallelization techniques on parallel computers that can perform distributed computations simultaneously on multiple processors. All the DE efficiency enhancement methods described here lend themselves to such parallelization in a straightforward manner. In the present work the various methods have been implemented on distributed parallel computers such as the SGI Origin 3000 . It is noted that most of the computing time is expended in the aerodynamic analyses. Parallel implementation relies on the simultaneous computation of multiple, independent aerodynamic simulations on separate processors. A script-based procedure is used that invokes a variable number of processors depending on processor availability and the population size. A "master-slave" arrangement is used, with the master handling the tasks of setting up the simulations, neural network training as needed, and the farming out of the aerodynamic computations to the other slave processors. Since the aerodynamic computations are independent of each other, no communication between the processors is required until the computations are completed. The slave processors then communicate their results to the master which then performs the necessary calculations to determine the population members of the next generation. In the injection island approach, an overall master assigns to each sub-population a master and a fixed number of slave processors; some additional communi- cation burden is incurred between the subpopulations during the migration phase.

\section{AERODYNAMIC SHAPE OPTIMIZATION METHOD IMPLEMENTATION DETAILS}

Some details regarding other aspects of the DE-based aerodynamic shape optimization method incorporating the various efficiency enhancement approaches are described briefly in this section.

\section{Constraint Handling}

An efficient constraint handling mechanism is incorporated in the optimization method. It is a parameterless approach that helps steer the algorithm away from infeasible regions of the design space. This constraint handling method was found to perform well when applied to various test problems taken from the constrained optimization literature. The constraint handling mechanism was adapted for use in aerodynamic optimization where both physical constraints (e.g. maximum airfoil thickness) as well as aerodynamic constraints (e.g. wavy surfaces) are imposed. Airfoil geometries that do not violate the constraints but for which the CFD solver fails to converge are also deemed infeasible and handled appropriately.

\section{Airfoil Geometry Parametrization}

Geometry parametrization and prudent selection of design variables are critical aspects of any shape optimization procedure. Since this study focuses on airfoil redesign, the ability to represent various airfoil geometries with a common set of geometrical parameters is essential. Variations of the airfoil geometry can be obtained then by smoothly varying these parameters. Geometrical constraints imposed for various reasons, such as structural, aerodynamic (e.g., to eliminate flow separation), etc., should be included in this parametric representation as much as possible. Additionally, the smallest number of parameters should be used to represent the family of airfoils. Here, the airfoil geometry parametrization method ${ }^{8}$ is adopted with a total of 13 geometric parameters being used to define the airfoil geometry. This parametrization provided the necessary variations in airfoil geometry required by the optimization procedure.

\section{CFD Simulation Methodology}

A Navier-Stokes solver was used to perform the flow simulations (direct function evaluations) that serve as inputs to the shape optimization process. The solver used is a modified version of the ROTOR-2 computer $\operatorname{code}^{23}$ and solves the two-dimensional, Navier-Stokes equations around a single airfoil in a cascade (with 
spanwise periodic boundary conditions) for a given set of inlet and exit conditions. Multiple grids are used to discretize the flow domain; an inner " 0 " grid that contains the airfoil and an outer " $\mathrm{H}$ " grid that conforms to the external boundaries as shown in Fig. 1. For the analyses performed here, each inner $O$ grid has 151 points in the circumferential direction and 41 points in the wallnormal direction. Each outer $\mathrm{H}$ grid has 101 points in the axial direction and 41 points in the transverse direction. For the sake of clarity, only some of the grid points are shown in Fig. 1.

The dependent variables are initialized to freestream valucs and the cquations of motion arc then integrated subject to the boundary conditions. The flow parameters that are specified are the pressure ratio across the turbine (ratio of exit static pressure to inlet total pressure), inlet temperature and flow angle, flow coefficient, and unit Reynolds number based on inlet conditions.

\section{Design Objective Formulation}

Various design objectives can be incorporated in the optimization method depending on the problem being solved. For the inverse turbine airfoil design shown here, the design objective function was formulated as the equally-weighted sum-of-squares error between the target and actual pressure obtained during the optimization process at various locations on the airfoil.

\section{RESULTS}

In the final paper, results obtained using the various approaches will be presented in this section. The approaches will be first tested on various unconstrained and constrained test problems taken from the optimization literature. For the test problems some effort was made to determine appropriate input parameter settings that resulted in good overall convergence. Results for aerodynamic shape optimization of a turbine airfoil will be then presented and compared using the various approaches. In order to provide a flavor of the results some prior results using a couple of the approaches described here are included. These results were presented the author in earlier work. ${ }^{8,9}$ The final paper will include more detailed comparisons between these and the other approaches described in this paper.

\section{Test Optimization Problem}

The test problem considered is the well-known Levy No. 5 function. ${ }^{24}$ The topology of this objective function $f_{1}$ (see Ref. 9 for details) is shown in Fig. 2. Note that the negative of the function is plotted so all the minima appear as maxima in the figure. There are about 760 local minima with one global minimum and the large number of local optima makes it difficult to locate the global minimum.

The performance of the memetic $\mathrm{DE}$ and baseline $\mathrm{DE}$ algorithms on this test problem is compared in Table 1. The reliability values in Table 1 denote the percentage of independent tests that converged to the correct optimum and are a measure of the robustness of the algorithms. On test problem $f_{1}$, the base $\mathrm{DE}$ algorithm required a population size of 25 and 2045 objective function evaluations to converge to the correct optimum with $100 \%$ reliability; the memetic DE algorithm achieves the same $100 \%$ reliability with only a population size of 5 and 598 function evaluations. With a popu lation size this small, the base $\mathrm{DE}$ algorithm is unable to converge with more than $30 \%$ reliability. Thus the memetic DE method is able to achieve a marked improvement of $70 \%$ in the number of function evaluations required for the functions $f_{1}$.

\begin{tabular}{|c|c|c|c|l|c|}
\hline $\begin{array}{l}\text { Test } \\
\text { Prob- } \\
\text { lem }\end{array}$ & $\begin{array}{l}\text { Algo- } \\
\text { rithm }\end{array}$ & $\begin{array}{l}\text { Pop. } \\
\text { Size }\end{array}$ & $\begin{array}{l}\text { Func- } \\
\text { tion+Con } \\
\text { straint } \\
\text { Evalua- } \\
\text { tions }\end{array}$ & $\begin{array}{l}\text { Func- } \\
\text { tion } \\
\text { Evalua- } \\
\text { tions }\end{array}$ & $\begin{array}{l}\text { Reli- } \\
\text { ability } \\
(\%)\end{array}$ \\
\hline $\mathrm{f}_{1}$ & $\mathrm{DE}$ & 5 & $\mathrm{n} / \mathrm{a}$ & 464 & 30 \\
\hline & $\mathrm{DE}$ & 10 & $\mathrm{n} / \mathrm{a}$ & 821 & 81 \\
\hline & $\mathrm{DE}$ & 15 & $\mathrm{n} / \mathrm{a}$ & 1256 & 98 \\
\hline & $\mathrm{DE}$ & 20 & $\mathrm{n} / \mathrm{a}$ & 1633 & 99 \\
\hline & $\mathrm{DE}$ & 25 & $\mathrm{n} / \mathrm{a}$ & 2045 & 100 \\
\hline & $\mathrm{DE}-\mathrm{DHC}$ & 5 & $\mathrm{n} / \mathrm{a}$ & 598 & 100 \\
\hline $\mathrm{f}_{3}$ & $\mathrm{DE}$ & 10 & 11302 & 7344 & 60 \\
\hline & $\mathrm{DE}$ & 15 & 15428 & 8614 & 90 \\
\hline & $\mathrm{DE}$ & 20 & 21694 & 11050 & 100 \\
\hline & $\mathrm{DE}-\mathrm{DHC}$ & 5 & 4689 & 2138 & 100 \\
\hline
\end{tabular}

Table 1. Performance comparison of $\mathrm{DE}$ and hybridized DE-DHC algorithms on unconstrained and constrained optimization test problems. Results based on an average of 100 ( 20 for $f_{3}$ case) independent test runs

Figure 3 compares the convergence behavior of the memetic and standard DE algorithms on the test function $f_{1}$. The ordinate in the figure is the objective function value for the best population member at each generation. Typical results are shown from sample test runs with population sizes for both algorithms chosen for $100 \%$ reliability. In both cases, the $\mathrm{DE}$ algorithm exhibits a typical step convergence pattern, where the objective function value remains constant for several generations before descending to the next lower level. Due to the small population size, the memetic DE starts at higher objective function values but is able to converge rapidly. On the other hand, DE shows good convergence behavior initially, but then slows down in later generations as the optimum is approached. 


\section{Aerodynamic Shape Optimization}

The standard $\mathrm{DE}$ algorithm and the various efficiency improving ideas were also were used in the inverse design of a turbine airfoil with a specified pressure distribution. Results ${ }^{9}$ are shown here for the baseline DE and the metamodel and memetic variants. The target pressure distribution was obtained at the midspan of a turbine vane from a modern Pratt and Whitney jet engine. Several flow and geometry parameters were also supplied and used in the design process. The design objective function was formulated as the equallyweighted sum-of-squares error between the target and actual pressure obtained during the optimizationi process at 45 locations on the airfoil.

The initial design space was chosen to be quite large to allow a wide range of airfoil shapes to be explored. A sampling of some of the initial airfoil geometries is shown in Fig. 4. In order to hold the CFD function evaluations to a reasonable number, 6 design variables (instead of 13) were used in the initial stages of the design. A population size of 25 and 10 members was used for the metamodel and memetic DE algorithms, respectively.

For the metamodel DE algorithm, several generations were first evolved using the base $\mathrm{DE}$ algorithm. In the early stages of evolution several of the airfoil geometries were determined to be infeasible and hence were not evaluated by the CFD solver. After 13 generations had evolved, the number of design variables was increased from 6 to 13 and the population was evolved further for another 5 generations. At this point the switch to the metamodel DE algorithm was made with the results from this generation being used to train the neural network. Figure 5 shows the data used in neural network training in the form of an envelope of pressure data around the target pressure distribution. Note, however, that the network was trained directly on the sum-squareerror and not on the individual pressure data. The data envelope in Fig. 5 represents the variation of the pressure distributions for the range of airfoil geometries in the neural network training set and is meant to convey the local nature of the neural network response surface in the vicinity of the optimal solution.

For the memetic DE algorithm, a small population size of 10 members was used. At each generation, the $\mathrm{CV}$ ratio of the population was monitored and the best member of the population was picked for DHC search if deemed necessary. In order to minimize the number of function evaluations required, the DHC search was constrained to a small local region around the chosen location in design space. The diversity-preserving techniques described earlier were used to ensure that the solution did not converge prematurely although this was not much of an issue for this particular objective function landscape.

The optimal pressure distributions obtained using the two DE variants are shown in Fig. 6. Both methods are seen to agree well with the target $P \& W$ distribution. The airfoil geometries corresponding to these optimal pressure distributions are also in close agreement as shown in Fig. 7.

The above represents some of the results obtained. The final paper will evaluate and compare the various approaches described on these and additional optimization problems. Detailed measurements of algorithm convergence will also be included.

\section{REFERENCES}

${ }^{1}$ Storn, R. and Price, K., "Differential Evolution - A Simple Evolution Strategy for Fast Optimization," Dr. Dobb's Journal, Vol. 22, No. 4, pp. 18-24, April 1997.

${ }^{2}$ K. V. Price: 'Differential Evolution: A Fast and Simple Numerical Optimizer'. In: Biennial Conference of the North American Fuzzy Information Processing Society, (NAFIPS), June 1996, ed. by M. Smith, M. Lee, J. Keller, J. Yen (IEEE Press, New York 1996) pp. 524-527.

${ }^{3}$ J. Lampinen: A Bibliography of Differential Evolution Algorithm. Technical Report. Lappeenranta University of Technology, Department of Information Technology, Laboratory of Information Processing, Lappeenranta, Finland (2001).

${ }^{4}$ Nho, K., and Agarwal, R. K., "Fuzzy Logic ModelBased Predictive Control of Aircraft Dynamics Using ANFIS," AIAA Paper 2001-0316, Jan., 2001.

${ }^{5}$ Rogalsky, T., Derksen, R.W. and Kocabiyik, S., "Differential Evolution in Aerodynamic Optimization," Canadian Aeronautics and Space Institute Journal, Vol. 46, No. 4, pp. 183-190, Dec. 2000.

${ }^{6}$ Rogalsky, T. and Derksen, R.W., "Hybridization of Differential Evolution for Aerodynamic Design," Proceedings of the 8th Annual Conference of the Computational Fluid Dynamics Society of Canada, pp. 729-736, June 11-13, 2000.

${ }^{7}$ Rai, M. M., "Towards a Hybrid Aerodynamic Design Procedure Based on Neural Networks and Evolutionary Methods," AIAA Paper No. 2002-3143. June 2002.

${ }^{8}$ Madavan, N. K., "Turbomachinery Airfoil Design Optimization Using Differential Evolution," 2nd International Conference on Computational Fluid Dynamics, Sydney, Australia, Jul. 2002. Lecture Notes in Physics, Springer-Verlag, to appear.

${ }^{9}$ Madavan, N. K., "Aerodynamic Shape Optimization Using Hybridized Differential Evolution," AIAA Paper 
No. 3792, AIAA Applied Aerodynamics Conference, Orlando, FL, June 24-27, 2003.

${ }^{10}$ Chiou, J., and Wang, F. S., "A Hybrid Method of Differential Evolution with Application to Optimal Control Problems of a Bioprocess System," Proceedings of the IEEE International Conference on Evolutionary Computation, IEEE, New York, NY, pp. 627-632, 1998.

${ }^{11}$ Back. T, Hammel, U., and Schwefel, H.-P., "Evolutionary Computation: Comments on the History and Current State," IEEE Trans. on Evolutionary Computation, Vol. 1, pp. 3-17, 1997.

${ }^{12}$ M. A. Shokrollahi, and R. Storn: 'Design of Efficient Erasure Codes with Differential Evolution'. In: Proceedings of ISIT 2000, International Symposium on Information Theory, Sorrento, Italy, June 25-30, 2000.

${ }^{13}$ Alexandrov, N. M., Dennis, J. E., Lewis. R. M., and Torczon, V., "A Trust Region Framework for Managing the Use of Approximation Models in Optimization," Structural Optimization, Vol 15, No. 1, pp. 16-23, 1998.

${ }^{14}$ Yuret, D., and Maza, M., "Dynamic Hill Climbing: Overcoming the Limitations of Optimization Techniques," 2nd Turkish Symposium on Artificial Intelligence and Neural Networks, pp. 208-212, 1993.

${ }^{15}$ Yuret, D., and Maza, M., "Dynamic Hill Climbing," AI Expert, Vol. 9,. No. 3, pp. 26-31, March 1994.

${ }^{16}$ Espinoza, F. P., Minsker, B. S., and Goldberg, D. E., "A Self-Adaptive Hybrid Genetic Algorithm," Proceedings of the Genetic and Evolutionary Computation Conference (GECCO-2001), International Society for Genetic and Evolutionary Computation, San Francisco, CA, 2001.

${ }^{17}$ Eby, D., Averill, R. C., Gelfand, B., and Punch, W., "An Injection Island GA for Flywheel Design Optimization," Eufit 1997, 5th European Congress on Intelligent Techniques, H. Zimmerman, Ed., Verlag, Aachen, pp. 687-691, 1997.

${ }^{18}$ Vekeria, H. D., and Parmee, I., "Cooperative Evolutionary Strategies for Single Component Design," in Proceedings of the 7th Int. Conf. on Genetic Algorithms, Back, T. Ed., Morgan Kaufmann, San Francisco, pp. 529-536, 1997.

${ }^{19}$ Schraudolph, N. N., and Belew, R. K., "Dynamic Parameter Encoding for Genetic Algorithms," Machine Learning, Vol. 9, pp. 9-21, 1992.

${ }^{20}$ Krishnakumar, K., Swaminathan, R., Garg, S., and Narayanaswamy, S., "Solving Large Parameter Optimization Problems Using Genetic Algorithms," Proc. of the Guidance, Navigation, and Control Conference, pp. 3136-3141, 1995.

${ }^{21}$ Oyama, A., Obayashi, S., and Nakahashi, K., "RealCoded Adaptive Range Genetic Algorithm and Its
Application to Aerodynamic Design," JSME International Journal, Series A, Vol. 43, No. 2, pp. 124-129, April 2000.

${ }^{22}$ Barthelemy, J-F., M., and Haftka, R. T., "Approximation Concepts for Optimum Structural Design -- A Review," Structural Optimization, Col. 5, pp. 129-144, 1993.

${ }^{23}$ Rai, M. M., and Madavan, N. K., "Multi-Airfoil Navier-Stokes Simulations of Turbine Rotor-Stator Interaction," ASME Journal of Turbomachinery, Vol. 112, pp. 167-190, Jul. 1990.

${ }^{24}$ Lëvy, A. V., Montalvo, A., Gomez, S., and Calderon, A., "Topics in Global Optimization," Lecture Notes in Mathematics, Springer-Verlag, Vol. 909, pp. 18-33, 1982.

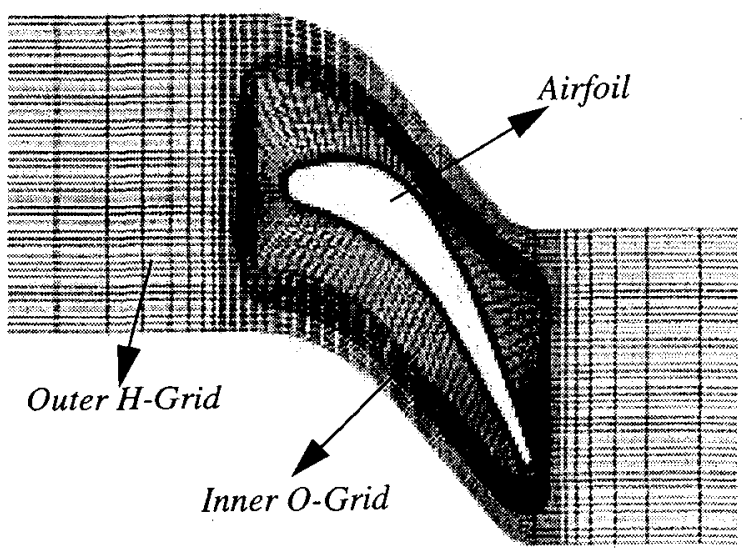

Figure 1. Representative turbine airfoil geometry and computational grid used in the CFD simulations. 


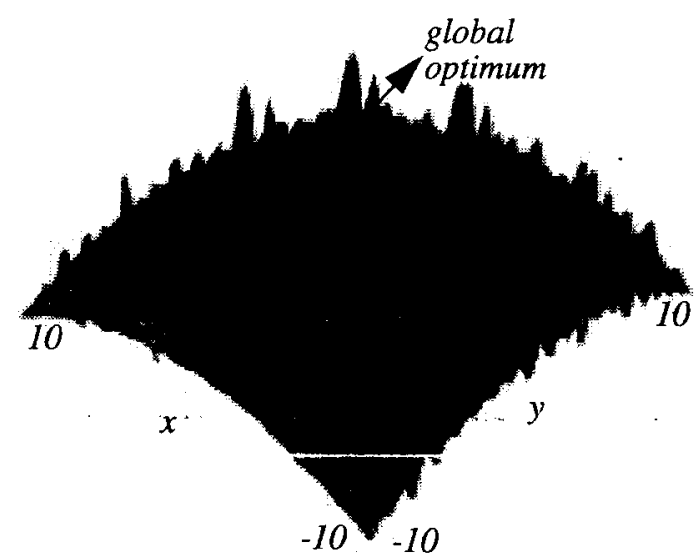

Figure 2. Topology of unconstrained test optimization function $f_{1}$ (Levy No. 5) in the search space.

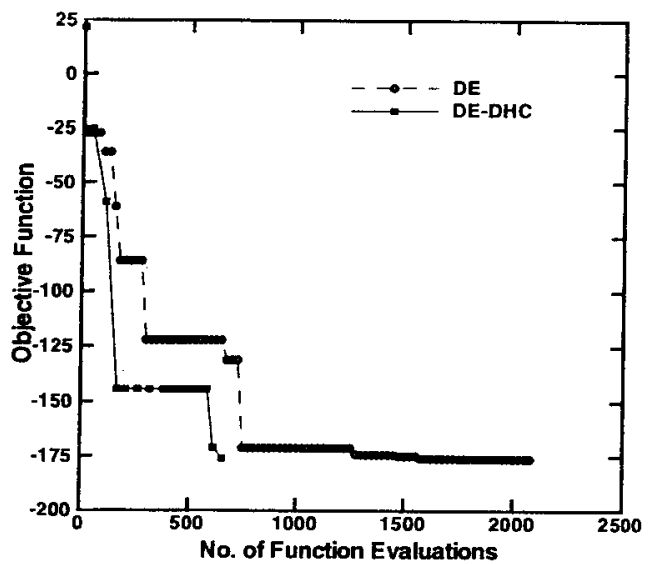

Figure 3. Comparision of convergence behavior of $\mathrm{DE}$ and memetic DE (DE-DHC) algorithms for function F1.

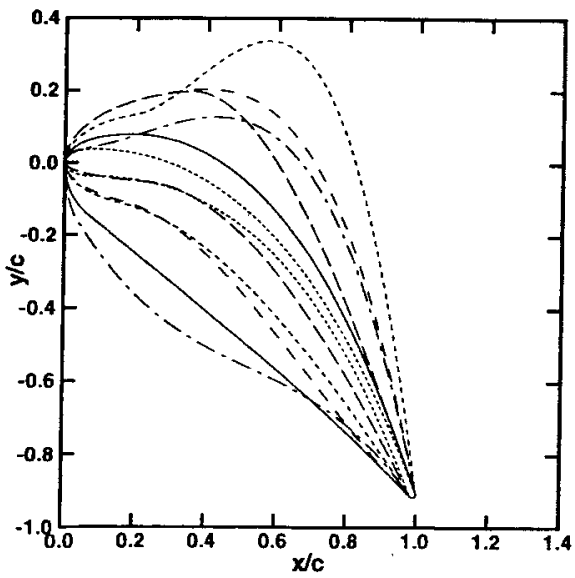

Figure 4. Sampling of initial airfoil geometries.

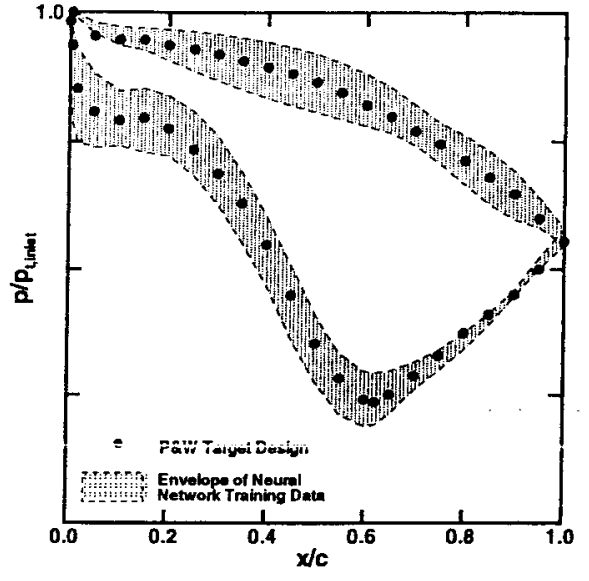

Figure 5. Envelope of airfoil loadings used to train the neural network for metamodel DE (DE-NN) algorithm.

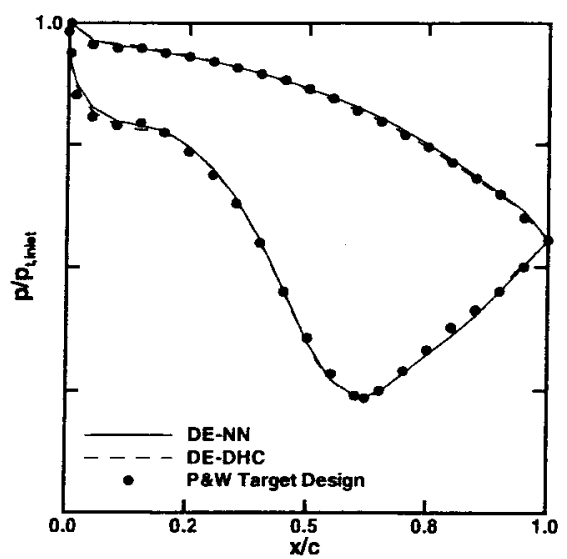

Figure 6. Airfoil pressure loading for the optimal design using DE variants.

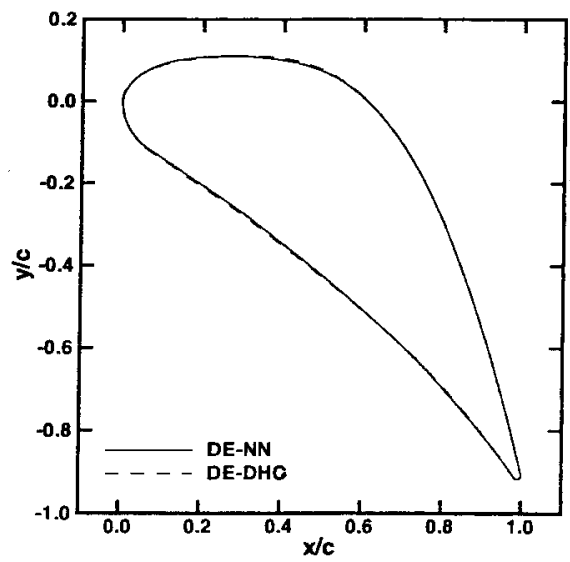

Figure 7. Airfoil geometry for the optimal design using $\mathrm{DE}$ variants. 